Int. J. Dev. Biol. 61: 95-104 (2017)

doi: $10.1387 / \mathrm{ijdb} .160038 \mathrm{ss}$

\title{
Dictyostelium discoideum Sir2D modulates cell-type specific gene expression and is involved in autophagy
}

\author{
RAKHEE LOHIA ${ }^{\#, 1,2}$, PUNITA JAIN,1,3, MUKUL JAIN"1, PRADEEP KUMAR BURMA ${ }^{2}$, \\ ANJU SHRIVASTAVA ${ }^{3}$ and SHWETA SARAN ${ }^{*, 1}$ \\ ${ }^{1}$ School of Life Sciences, Jawaharlal Nehru University, ${ }^{2}$ Department of Genetics, University of Delhi, South Campus \\ and ${ }^{3}$ Department of Zoology, University of Delhi, New Delhi, India
}

\begin{abstract}
Sirtuins (SIRTs) belong to class III histone deacetylases and require NAD+ for their activity. Their activity is associated with the nutritional status of the cell and they directly link cellular metabolic signalling to the state of protein post-translational modifications. Sirtuins play an important role in healthy aging, longevity and age-related diseases, as well as in cell survival mechanisms, such as autophagy. Here, we investigate the functions of Dictyostelium discoideum Sir2D which shows similarity to human SIRT1. This gene is expressed throughout growth and development. Overexpression of sir2D promotes cell proliferation and the corresponding fusion protein shows nuclear localization. To facilitate the study of the function of Sir2D, we created a sir2D knockout by gene disruption. This mutant exhibits inhibited cell proliferation and developmental defects, including smaller aggregates and multi-tipped structures. When developed as chimeras with wild-type cells, the sir2D cells show a reduced ability to form spores. Prespore and prestalk differentiation was also impaired in the mutant strain. Sir2D regulates the expression of several autophagic genes (Atgs) and the sir2D deficient strain shows reduced autophagic flux. In conclusion, Sir2D plays a role in cell differentiation, modulates the expression of both prespore and prestalk genes and participates in the process of autophagy.
\end{abstract}

KEY WORDS: Dictyostelium, Sir2D, development, cAMP, autophagy

\section{Introduction}

Sirtuins (SIRTs) are evolutionarily conserved class III histone deacetylases that are dependent on $\mathrm{NAD}^{+}$for its deacetylate activity. Since the discovery of Sir2 (silencing information regulator 2) in Saccharomyces cerevisiae as a transcriptional silencer of the mating-type loci many studies have been undertakento demonstrate the diverse biological roles, like cellular metabolism, lifespan regulation and genome stability (McBurney 2003; Guarente 2000). Each of the seven isoforms found in humans (SIRT1-7) have a unique subcellular localization and distinct functions (Finkel et al., 2009; Vassilopoulos et al., 2011). Sirtuins deacetylate various substrate proteins like histones and non-histones (Martínez-Redondo and Vaquero 2013) including transcription factors and metabolic enzymes that regulate various biological processes, like transcription, cell survival, DNA damage and repair, longevity etc. (reviewed by Dali-Youcef et al., 2007). Sirtuins are NAD-dependent and play a crucial role in modulating energy metabolism and also as important regulators of various metabolic pathways (Li and Kazgan 2011). They act as sensors of both energy and redox status of cells. Several sirtuins play roles in metabolic homeostasis, for example both SIRT1 and SIRT2 control autophagy responses under nutrient stress conditions (Nakagawa and Guarente 2011). It regulates autophagy through the deacetylation of autophagy-related genes (Atgs) and other mediators of autophagy. SIRT1 is shown to directly interact and deacetylate several Atg proteins like Atg5, Atg7, and Atg8 leading to its activation (Lee et al., 2008).

Autophagy is an evolutionary conserved pathway maintaining cellular homeostasis through removal and recycling of damaged organelles and macromolecules (He and Klionsky 2009). During autophagy, cytoplasmic materials are sequestered within a double-

Abbreviations used in this paper: Atg, autophagic gene; Sir, silencing information regulator; SIRT, sirtuin.

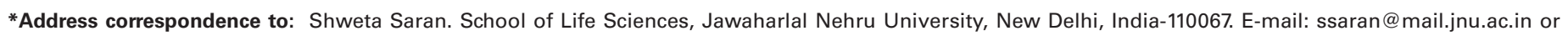
shweta_saran@hotmail.com - \# Note: Equal authorship.
}

Supplementary Material (five figures, one table) for this paper is available at: http://dx.doi.org/10.1387/ijdb.160038ss

Submitted: 8 February, 2016; Accepted: 23 June, 2016. 
SCHST1

HsSIRT1

Ddsir2D

ScSir2

ScHST1

Fig. 1. DdSir2D shares sequence homology with human SIRT1. Multiple sequence alignment of the core domain sequence of Sir2D from $D$. discoideum and other known homologs using ClustalW2. See text for details.

membrane vesicle called autophagosomes, which later fuse with the lysosome to form autolysosome and ultimately lead to the degradation of materials and subsequent recycling (Calvo-Garrido et al., 2010). It involves several sequential steps including autophagosome nucleation, elongation, lipidation and degradation that are controlled by Atgs (Calvo-Garrido et al., 2010). Protein kinase Atg1 and lipid kinase Vps34 and Atg13 are necessary for induction and nucleation of autophagososmes. The vesicle expansion and completion require Atg8 and Atg 12 while other proteins like Atg2, Atg9 and Atg18 help in membrane trafficking and biogenesis of autophagosomes. Many of these proteins are conserved in evolution and also found in D. discoideum (Calvo-Garrido et al., 2010). Autophagy plays an important role in differentiation and development of multicellular eukaryotes and the same is true for $D$. discoideum. Previously it has been shown that Atg1, Atg5, Atg6, Atg7, Atg8, Atg9 of vmp1 caused aberrant development with varying severity (Tung et al., 2010; Otto et al., 2003; Otto et al., 2004; Calvo-Garrido et al., 2010) in D. discoideum.

Dictyostelium discoideumbelongs to a group of unicellular life forms that can also exist as multicellular structures that depend on autophagy. $D$. discoideum amoebae grow and divide mitotically when food is sufficient but upon starvation and in response to the chemoattractant cAMP (Konijn 1961), they aggregate to common collecting points and undergo various morphogenetic movements to ultimately form a fruiting body comprising of two terminally differentiated cell types: the stalk (dead, vacuolated) and spore (viable) cells. The formations of stalk cells show signs of autophagy and excessive autophagy results in autophagic cell death (ACD) (Kessin 2001; Giusti et al., 2009). Multicellular development, which takes place in the absence of exogenous nutrients, requires mobilizing resources by glycogenolysis and autophagy. Induction and regulation of autophagy is tightly regulated by energy and nutritional status of the cell. Dictyostelium genome codes almost all the basic components required for regulating autophagy in other organisms (Calvo-Garrido et al., 2010). The strong similarity with animals and the presence of conserved proteins in $D$. discoideum and human shows high level of conservation of basic autophagy machinery shared between D. discoideum and human (Calvo-Garrido et al., 2010).

According to dictyBase (www.dictybase.org/), there are five sirtuins present in this organism. Presently, we have analyzed the D. discoideum SIRT1 ortholog (DdSir2D) by expressing as an Eyfp fusion protein and generating knockout cells. DdSir2D is localized to the nucleus and expressed throughout growth and development. Deletion leads to defects in cell proliferation, development and differentiation. Our results also show that deletion of Ddsir2D decrease the autophagic flux.

\section{Results}

\section{Putative Sir2D of D. discoideum shows homology to known sirtuins}

The $D$. discoideum genome, examined via the dictyBase contains a single gene (DDB_G0289967) with sequence similarity (48\%) to
A

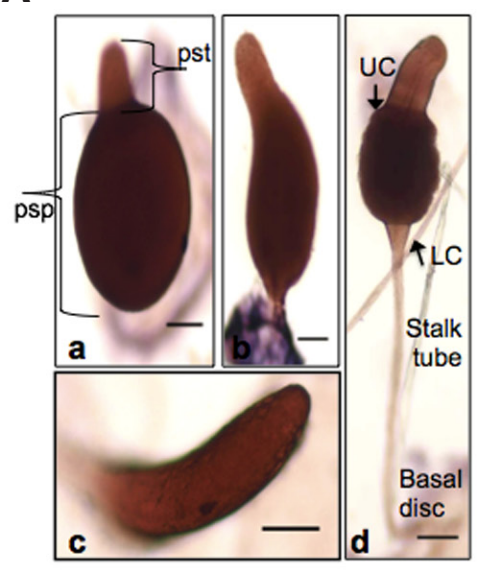

B

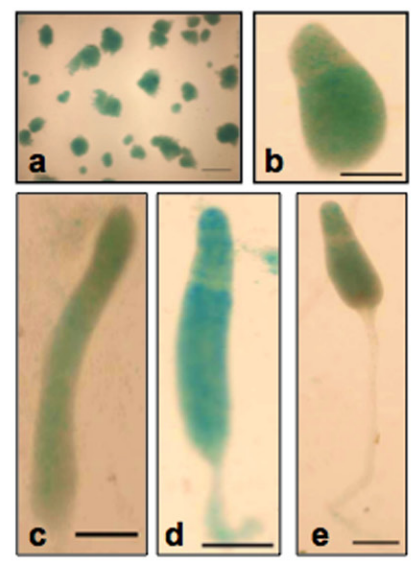

C

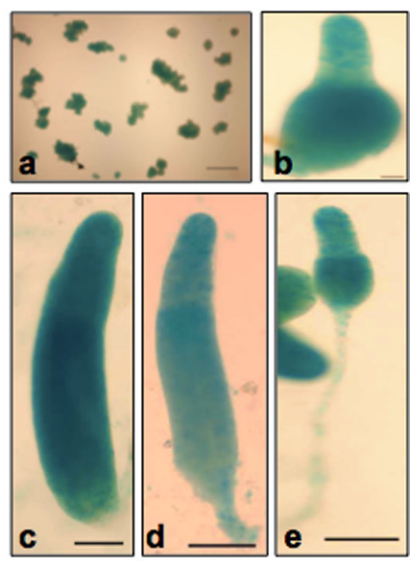

Fig. 2. Developmental regulation of sir2D transcript and protein in developing structures. (A) Spatiotemporal expression patterns as studied by in situ hybridization with Ddsir2D anti-sense probe. (B) sir2D promoter activity in wild-type cells transformed with vector [sir2D/ubilacZ] containing a gene fusion of the sir2D promoter and $\beta$-galactosidase (labile) reporter gene. (C) Sir2D fusion protein (ORF fused with $\beta$ galactosidase (labile) reporter gene) driven under its own promoter. Cells were developed, fixed and stained

with X-gal in multicellular structures (a-e). Bar length is 500 um. [a-tight-aggregates; b-first-finger; c-migrating slug; d-early-culminant; e-late-culminant; tight aggregates not shown in case of in situ hybridization results. Abbreviations: LC, lower cup; psp, prespore; pst, prestalk; UC, upper cup. 
human SIRT1. DdSir2D has a long $\mathrm{N}$ - terminal region with 5 low complexity regions and one coiled-coil region. Low complexity regions are present in both yeast Sir2 (ScSir2) and human SIRT1 (HsSIRT1), except that they are fewer in numbers. These regions are generally responsible for the divergence and compositional plasticity across protein families (Coletta et al., 2010).

When both full length and core domain protein sequences of DdSir2D were used as a query for BLASTp at NCBI it showed maximum homology to the HsSIRT1 (Supplementary Fig. S1). Sequence similarity of the conserved catalytic domain suggests the putative $D$. discoideum protein could be functionally similar to the HsSIRT1. We performed a multiple sequence alignment of the core domain of DdSir2D homologs from different organisms using ClustalW2 at EBI server to check for the conserved residues, which possibly could be responsible for its activity (Fig. 1). ClustalW2 results revealed the conserved sequences from HsSIRT1, ScSir2, Hst1 and DdSir2D, all of which belong to class la sirtuins (Frye 2000). In $D$. discoideum, the signature sequence GAGISXXXGIPXXR read as GAGvSVSCGIPDFR, where v (marked with small letter) shows an additional deviation from the original sequence (Frye 2000). This variation is also observed in HsSIRT1, ScSir2 and Hst1. Another signature sequence, $\mathrm{PXXXH}$ read as $\mathrm{PSPTH}$ in DdSir2D whereas PSLCH in HsSIRT1 and YSPLH in ScSir2 and Hst1. The HG motif essential for ADP-ribosylation is also present. Presence of $C$ before this HG motif belongs to class la i.e. CHG, in case of DdSir2D.

\section{sir2D is expressed throughout $D$. discoideum growth and development}

The temporal mRNA expression pattern of sir2D during growth and development was studied by RT-PCR (Supplementary Fig. S2). The patterns observed by us fits well with the developmental and cell-type specific mRNA expression data acquired by the microarray analysis, freely available in dictyBase.

Here, the spatial and temporal expression pattern of sir2D mRNA was analysed in developing multicellular structures by in situ hybridization (Fig. 2A). We found the levels to be comparatively more in the prespore cells except during the migrating slug stage (Fig. 2Ac) where the expression was almost equal in both the cell types. The spore cells of the culminant showed higher level of expression when compared to the stalk cells. Results obtained with sense probe are not shown.

The intergenic sequence upstream of the Ddsir2Dgene (hosting the putative promoter) along with the first 12 bases of the coding region were fused to the coding region of a rapidly degrading (labile) version of $\beta$-galactosidase and the resulting construct ([sir2/

Fig. 3. Subcellular localization and comparative analysis of growth and development. (A) Subcellular localization of Sir2D-Eyfp [Blue due to DAPI in nucleus; green is fusion protein]. a: DIC image; b: DAPI stain; c: Sir2D-GFP fusion protein and $d$ : merged image. (B) One representative experiment showing cell proliferation of wild-type and sir2D mutants. The generation time for each strain is also shown. (C) Analysis of development of various strains. Equal number of mid-log phase culture cells were plated and synchronized for development. The representative developmental stages are shown. Top panelshows the aggregate size, middle panel shows the slugs and lower panel shows the fruiting body formed. A minimum of 5-7 such areas per experiment was considered. Abbreviations: Ax2, wild-type; $O E$, sir2D ${ }^{\mathrm{OE}} ; \mathrm{KO}$, knockout; $R \mathrm{l}$, random integrant; sir2D- rescue, knockout strain transformed with plasmid used for overexpression. $n=4$; scale bar, $500 \mu \mathrm{m}$.
ubi-lacZ]) was transformed into Ax2 cells to examine the spatial expression patterns (Fig. 2B). As observed earlier, expression in tight aggregates (Fig. $2 \mathrm{Ba}$ ) was high. sir2D expression was almost equal in both the cell types in migrating slugs (Fig. 2Bc). In other multicellular structures developed (Fig. 2Bb, $d$ and e), lower expression was observed in prestalk region. Expression was observed in tip region of prestalk cells, basal disc and spore region of the early culminants (Fig. $2 \mathrm{Bd}$ ) while in fruiting bodies (Fig. 2Be) negligible expression was observed in the stalk region. Expression of sir2D was seen in the anterior prestalk cells but not in the lower cup region of the fruiting body. In order to circumvent the lack of an antibody the Ddsir2D gene was tagged to a rapidly degrading (labile) version of $\beta$-galactosidase and expressed in Ax2 cells ([sir2D/sir2D-ubi-lacZ]/Ax2) to indirectly visualize the spatial localization of the Sir2D protein in multicellular structures developed (Fig. 2C). The results obtained was similar to that obtained in Fig. 2B.

A

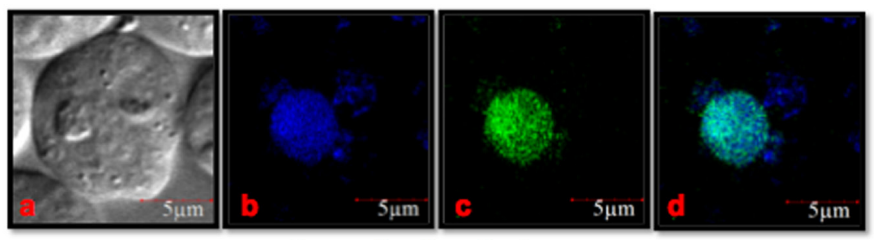

B

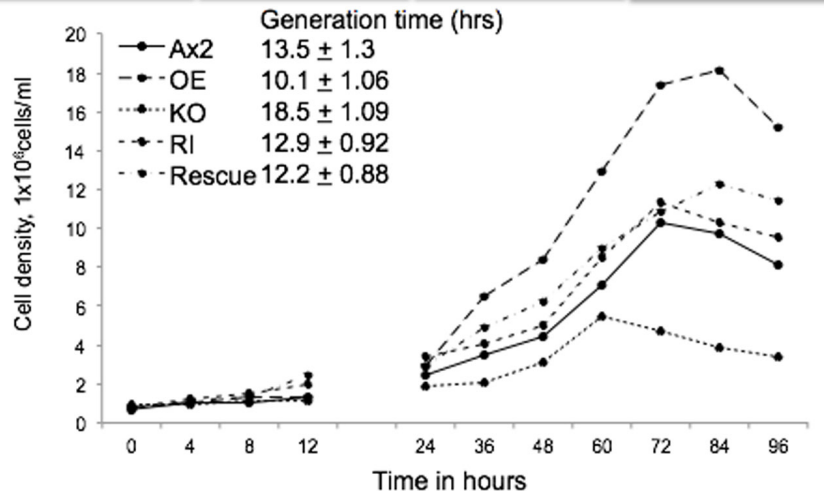

C

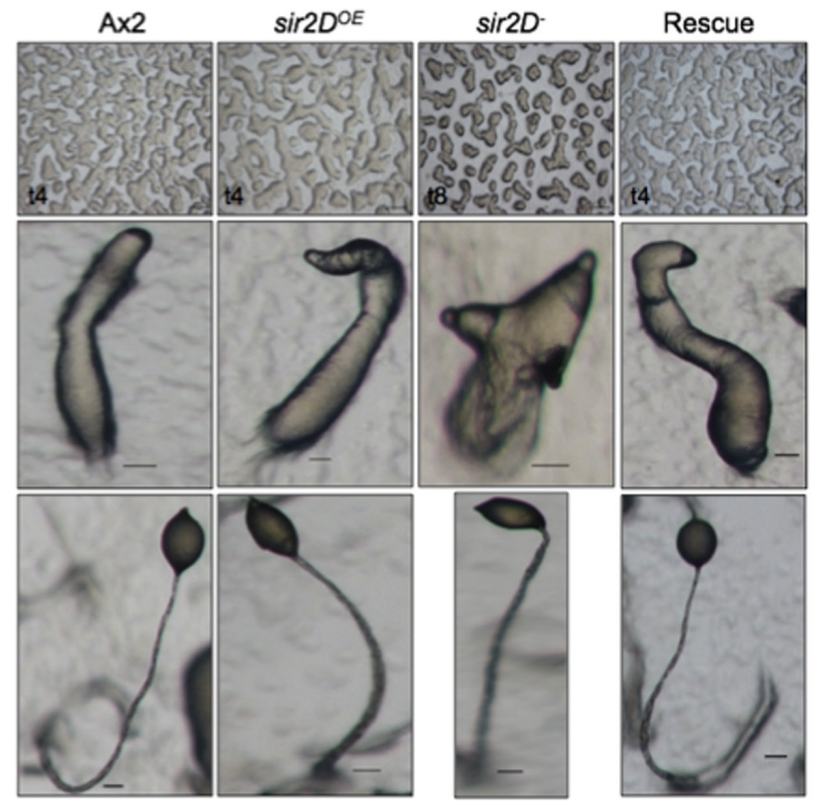




\section{Successful isolation of a sir2D - mutant}

To explore the DdSir2D function, we utilized the homologous recombination to create Dictyostelium strains carrying a disruption of the sir2D gene by the blasticidin resistance (Bsr) selection cassette. The strategy undertaken, confirmation of the mutants by PCR amplifications and sirtuin activity measurements are shown Supplementary Fig. S3. We could successfully isolate two independent strains each of knockout (KO), random-integrants (RI) and rescue (Res). Since both the strains for the given mutants gave similar results we proceeded with one of them for subsequent experiments.

\section{Sir2D fusion protein shows nuclear localization}

The subcellular localization of $D d s i r 2 D$ gene product as analysed by confocal microscopy (Fig. 3A) using [act15/sir2D-Eyfp/ Ax2] (Sir2 ${ }^{\circ}$ ) cells showed the expression to be exclusively in the nucleus. Expression of Sir2D showed same localization as their ortholog from humans (Michishita et al., 2005).

\section{Lack of Ddsir2D results in inhibition of cell proliferation and caused developmental defects}

To determine the role of Sir2D in cell proliferation we compared growth profiles of sir $2 D^{\circ E}$, sir $2 D^{-}, \mathrm{Rl}$ and Res strains to the wild-type Ax2. We grew all the strains under similar conditions and counted them in a haemocytometer for 96 hours (Fig. 3B). Lack of Sir2D inhibited cell proliferation and showed a longer lag phase (till $t_{12}$ ) as compared to other strains under study. Also, stationary phase reached by $t_{60}$ and was less than $5 \times 10^{6} \mathrm{cells} / \mathrm{ml}$ as opposed to wild-type which showed maximum density of $\sim 1.2 \times 10^{7} \mathrm{cell} / \mathrm{ml}$ at $t_{72}$. The generation time in the logarithmic growth phase was found to be approximately $60 \%$ longer than the Ax 2 cells. On the other hand, Sir2 $\mathrm{D}^{\mathrm{OE}}$ showed high cell proliferation with maximum density of $\sim 1.8 \times 10^{7} \mathrm{cell} / \mathrm{ml}$ with a sharp increase in cell number. $\mathrm{Rl}$ and Res strains were similar to wild-type.

To determine whether the mutants of DdSir2D were capable of carrying out multicellular development, we assayed the different developmental stages in sir2D mutant strains and compared them to wild-type. Wild-type (Ax2), rescue (sir2D-Eyfp/sir2D) and sir2 $D^{O E}$ strains were comparable in their developmental timings, numbers and morphologies of multicellular structures formed during development. The sir2 $D^{-}$cells exhibited morphological defects when starved and plated for development. They started to aggregate later than the wild-type and the territory size of the aggregates was smaller. Multiple tips (nearly 3-4) appeared for each mound within cluster and these then later on formed normal sized and mostly individual slugs and fruiting bodies with shorter than normal stalks. Few of the fruiting bodies showed thickened stalk. Fig. $3 \mathrm{C}$ shows few representative developmental stages of

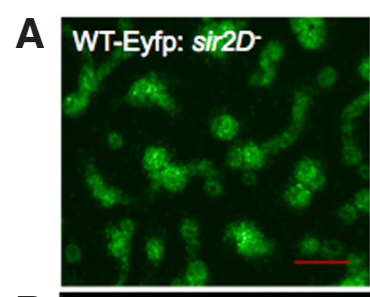

B
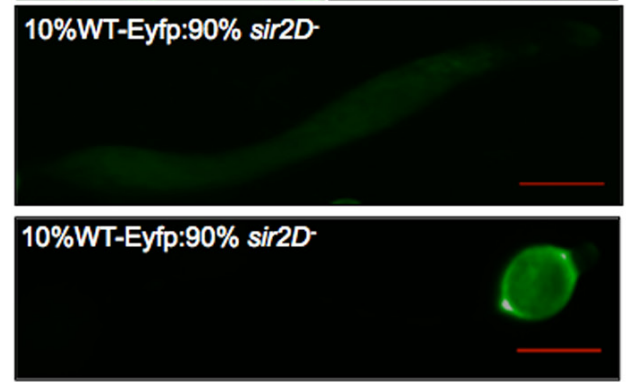

C
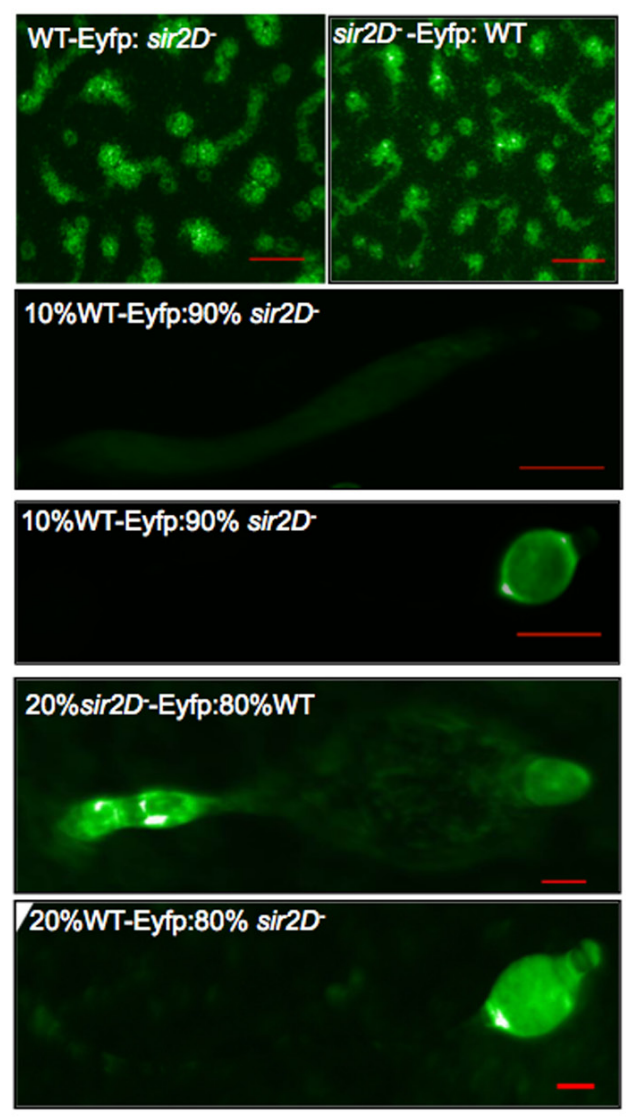

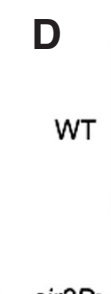

$20 \%$ act15-Eyfp V $80 \%$ unmarked

$\operatorname{sir} 2 D$

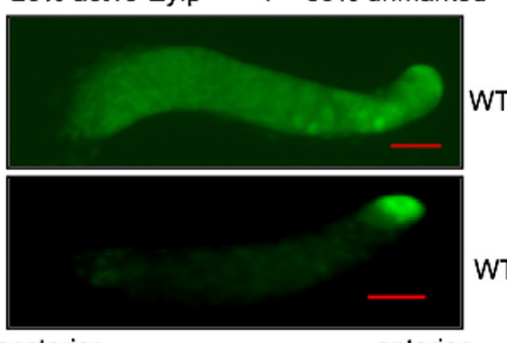

Eir2D-

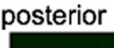

anterior

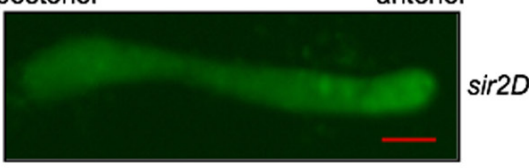

W

$\mathbf{F}$

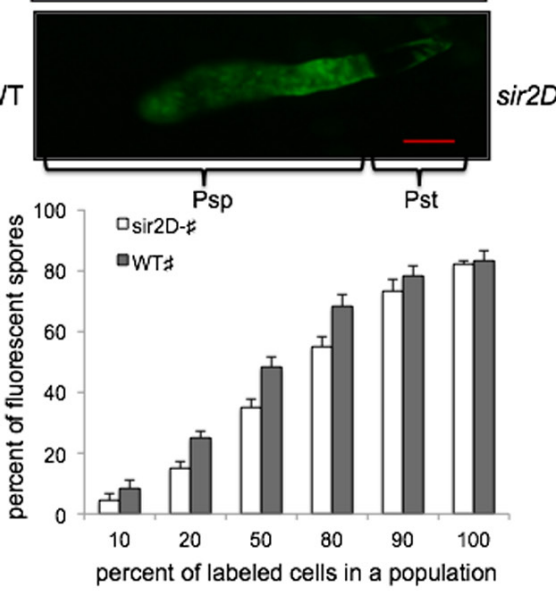

Fig. 4. Synergy experiments with wildtype and sir2D-null cells. (A) Eyfp was expressed in wild-type or sir2D- cells and their position in chimeric aggregation territories with unlabelled sir2D or wild-type cells, respectively was monitored. Mixed (1:1) cell suspensions were developed on non-nutrient agar plates and aggregation centres were imaged both under brightfield and fluorescence. Both wild-type and mutant showed equal probability in forming the aggregate centres (B) Chimeras with $10 \%$ labelled Ax2 cells and 90\% unlabelled sir2D cells showed the wild-type to sort to prespore region (upper panel) in slugs and sorus (lower panel) in fruiting body. The wild-type cells were able to rescue the phenotype of the mutant cells. (C) $20 \%$ labelled sir2D cells were mixed with $80 \%$ unlabelled wild-type cells (upper panel) and allowed to develop. The mutant cells sorted to the stalk and the upper cup region. In the vice-versa experiment (lower panel) the labelled wild-type cells sorted to the spore region. (D) 20\% labelled wild-type cells when mixed with 80\% unlabelled wild-type showed the labelled cells sorted to the entire structure (upper panel). But when 20\% unlabelled mutant cells were mixed with $80 \%$ unlabelled wild-type the mutant cells sorted to the anterior prestalk region (lower panel). (E) In the vice-versa experiment, the labelled mutant cells sorted to the

entire structure formed when mixed with its own unlabelled genotype (upper panel). The labelled wild-type cells sorted to the prespore region largely (lower panel) (F) Graph showing the fluorescent spores formed by the labelled mutant or wild-type cells at different ratios. Results are representative of four independent experiments for all panels shown. Nearly 20 spore-heads per experiment for each ratio was scored and an average was calculated. "Shows marked cells in the chimeras. Images are representative of three independent experiments. $n=4$; scale bar, $500 \mu \mathrm{m}$. 
the strains studied. The fusion protein is functionally active was confirmed by the near normal phenotype obtained by the rescue strain. In the wild-type, we observed loose aggregates by $4 \mathrm{~h}$ of starvation, which went on to form mounds by 8-10 h, slugs by 12-16 $\mathrm{h}$, early culminant by 18-20 $\mathrm{h}$ and fruiting bodies by $24 \mathrm{~h}$ as expected. The number of multicellular structures developed in a given unit area for each strain was also calculated (data not shown). A minimum of 7-10 such areas for each experiment was observed. We found less multicellular structures formed when sir2D cells were plated for development. Results clearly indicate that the Sir2D could possibly be involved in autophagy as the phenotypes shown were similar to that observed with mutants of core autophagy genes of $D$. discoideum.

\section{Wild-type and sir2D - cells do not distribute to all cell types when co-developed}

Differentiation preferences of mutant cells can be deduced from the sorting patterns of mutant cells when co-developed with wild-type cells. For this, mutant sir2 $D^{-}$and wild-type cells were labelled with an act15/Eyfp construct and mixed in varying ratios (from 10-90\%) with unmarked cells of the opposite genotype (or the same genotype as controls) (Fig. 4). We studied the contributions by lineage tracing of Eyfp-marked cells during slug and terminal differentiation of chimeras developed. During normal development of wild-type cells, approximately $80-85 \%$ of the total population forms spores and the rest contributes to stalk cells. We traced the cellpattern organization in live cells during slug stage where prestalk cells largely populate the anterior region and the prespore cells occupy the posterior region and in the fruiting body. The number of fluorescent spores in each spore head was counted.

A minimum of $10 \%$ wild-type cells could rescue the observed phenotype suggesting that the defects observed during the development of sir2D cells can be corrected during chimera formation (Fig 4B). The null cells sorted preferentially to the prestalk/stalk cells. The size and number of aggregates formed was similar to that observed with the wild-type cells alone. When 1:1 ratio of cells were plated, aggregates formed were contributed by both the mutant as well as the wild-type cells and either could form the centre of the aggregate (Fig. 4A).

When $20 \%$ of the marked sir2D cells were mixed with $80 \%$ of wild-type cells and developed under standard conditions, we found the null cells to sort to the prestalk region. Marked cells were observed in the prestalk region, rear-guard cells and ALCs of the slugs formed (Fig. 4D lower panel). During culmination, the sir2D cells specifically sorted to the upper cup but not to the lower cup, stalk but not to the basal disc (Fig. 4C upper panel). In the reciprocal combination (20\% labelled Ax2 cells mixed with unlabelled $80 \%$ sir2D null cells), the Ax2 cells moved to the prespore region and to the anterior-most tip region (Fig. 4E lower panel). In the culminant, the Ax2 cells were found in the spore mass and to a lesser extent in the tips (Fig. 4C lower panel).

When labelled (20\%) and unlabelled (80\%) cells of the same genotype were mixed and developed they formed normal slugs and culminants with fluorescence distributed throughout the structures developed (Fig. 4D upper panel and Fig. 4E upper panel for wildtype and sir2D-cells, respectively). Fig. 4F shows a comparison of spore forming tendencies by different strains when mixed in varying ratios. Our results show lower spore forming tendency by the mutant cells in comparison to the wild-type cells. We could conclude that in the chimeras developed with wild-type and sir2 $D^{-}$cells the null cells showed a preference for the prestalk/stalk cells and have a lower tendency to form spores.

\section{Lack of sir2D and aberrant cell-type patterning}

During development of $D$. discoideum, cells terminally differentiate into two main cell types, the stalk cells and the spore cells whose precursors namely prestalk and prespore respectively, can be observed at earlier developmental stages and is distinguished by marker genes. We have used these specific marker genes to determine if the pattern and localization of cell types are disrupted in the sir2D cells (Fig. 5). To analyse the spatial expression patterns of different genes in the [sir2D/Ax2] strain, a clone of the mutant and the wild-type Ax2 cells were transformed with a lacZ reporter gene under the control of either the ecmB/ecmAO/pspA promoters.

The prestalk specific ecmB promoter that is active in spe-

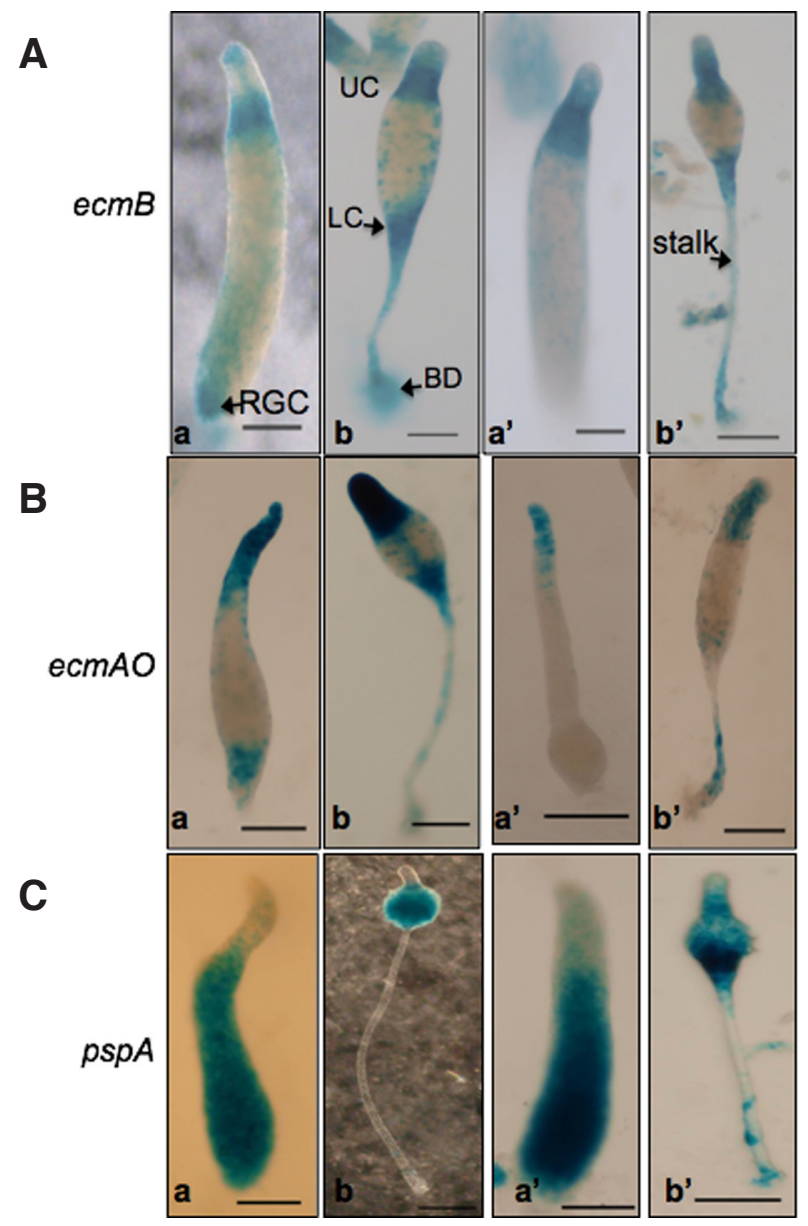

Fig. 5. Distribution of prestalk and prespore cells in sir2D mutant developmental structures. Wild-type and sir2D cells were transfected with reporter vectors where lacZ was expressed under the control of the promoter region from the prestalk-specific genes. (A) ecmB- [ecmB-lacZ], (B) ecmAO- [ecmAO-lacZ] or the (C) prespore-specific gene pspA- [pspAlacZ]. Pools of transfected cells were placed on nitrocellulose filters to induce multicellular development. Structures were collected at the slug and culminant stages of development, histochemical X-gal staining and photographed determined lacZ expression. $n=4$; scale bar, $500 \mu \mathrm{m} ; B D$, basal disc; $L C$, lower cup; $R G C$, rear-guard cell; UC, upper cup. 
cific prestalk cells including pstB and pstAB cells was expressed in the anterior one-fifth, anterior-like cells (ALCs) and the rear-guard cells (RGC) of the wild-type slugs. Expression was seen in the upper and lower cups, stalk-tube and basal disc region in the fruiting body of wild-type (Fig. $5 \mathrm{~A} a$ and $\mathrm{b}$ ). In the sir2 $\mathrm{D}$ slugs and fruiting bodies formed (Fig. 5A a' and b'), we observed insignificant differences both in localization and level of expression. The prestalk specific ecmAO promoter that is active in both pst $A$ and pstO cells is expressed in the anterior of slugs (Fig. 5Ba and b) was observed to be reduced in the sir2 $D^{-}$slugs and fruiting bodies formed (Fig. 5Ba' and b'). Prespore specific promoter $p s p A$, express in the posterior four-fifth of the slug and in the spore mass of the fruiting bodies formed in wild-type (Fig. $5 \mathrm{Ca}$ and b). In sir2D- slugs (Fig. $5 \mathrm{Ca}^{\prime}$ ) the

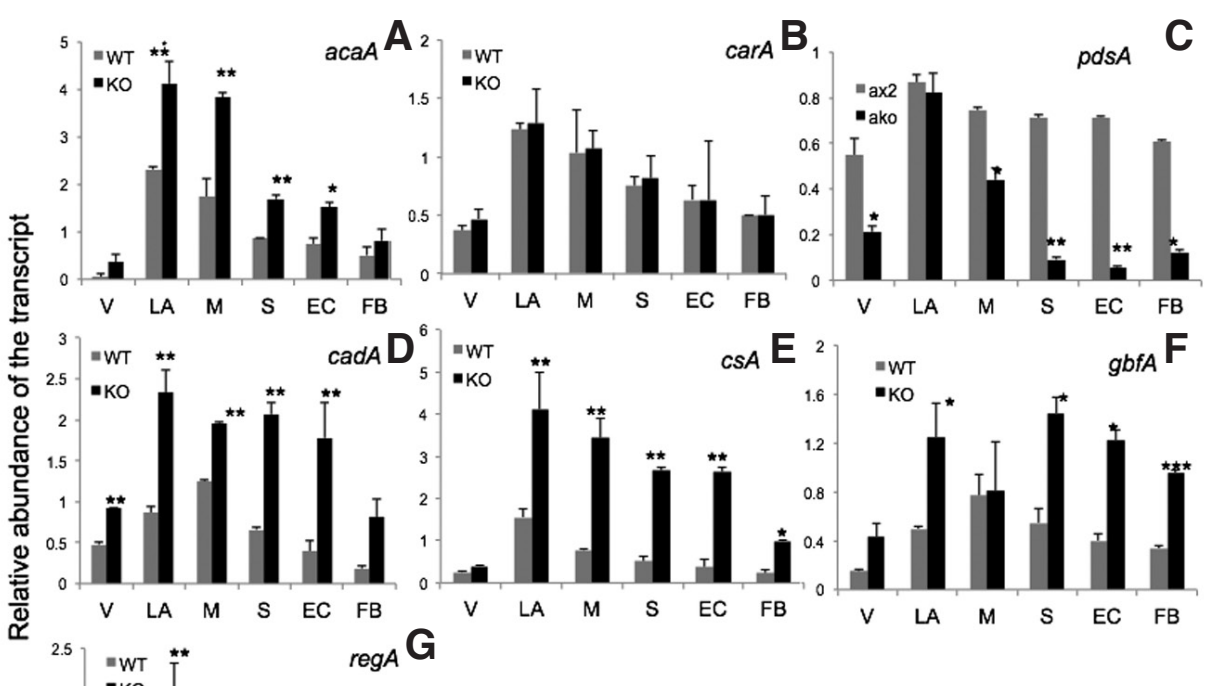
expression was reduced and also the demarcation between the prestalk and prespore was lost. We observed low expression in the prestalk region. In the fruiting bodies formed with sir2 $D^{-}$cells (Fig. $5 C^{\prime}$ 'and $^{\prime}$ '), similar results were obtained. We found activity in the stalk tube and lower cup regions. The expression was low in the spore mass. A comparison of the transcript levels for the ecmA, ecmB and $p s p A$ genes during development in both wild-type and mutant cells (Supplementary Fig. S4) confirmed our results.

Since the results obtained showed aberration in the $p s p A$ and ecmAO promoter activity we further characterized the expression of few specific genes involved in cAMP signalling. The expression of acaA (adenylyl cyclaseA) (Fig. 6A) increased remarkably in the sir2D while the carA (cAMP receptorA) (Fig. 6B) showed insignificant increase over the wild-type. The level of $p d s A$ (extracellular phosphodiesteraseA) (Fig. 6C) decreased suggesting an increase in the overall cAMP signalling during early development. Both $\operatorname{cad} A$ (calcium-dependent cell-adhesion) (Fig.6D) and CSA (calcium-independent cell-adhesion) (Fig. 6E) expression significantly increased at all stages of development in the sir2D cells as compared to the wild-type. The gbfA

Fig. 7. Sir2D regulates the expression of autophagy-related genes. mRNA expression relative to the internal control ig7 during specific stages of development was analyzed by RT-PCR in wild-type ana sir2D cells. (A) Atg1; (B) Atg8; (C) Atg5; (D) Atg16; (E) Atg9; (F) Atg18 IV-freshly starved vegetative cells; LA-loose-aggregate; M-mound; S-migrating slug: EC-earlyculminant; FB-fruiting body;]. [ $n=4 ; p$ value: ${ }^{* * *}<0.001 ; * *<0.01 ; * 0.05$ ].

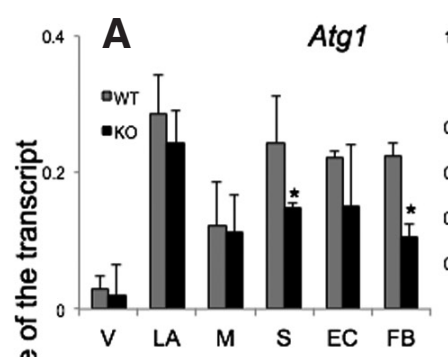

( $G$ protein binding factor) (Fig. 6F) and regA (intracellular phosphodiesterase) (Fig. 6G) expression was higher in the knockout strain. Results suggest increased CAMP signalling during early development but an increase in the regA transcript level suggests that CAMP signalling may decrease during later development so that normal development could proceed. Since absolute cAMP levels were not measured we cannot discuss this more.

\section{Sir2D regulates autophagy-related gene expression}

Autophagy is required for multicellular development in $D$. discoideum and the severity of the phenotype depends on the mutated genes. The multi-tipped phenotype of the sir2D cells allowed us to believe that Sir2D could be involved in autophagy. Thus, we asked if autophagy gene expression was regulated in the sir2D cells. Genes involved in the process of autophagy
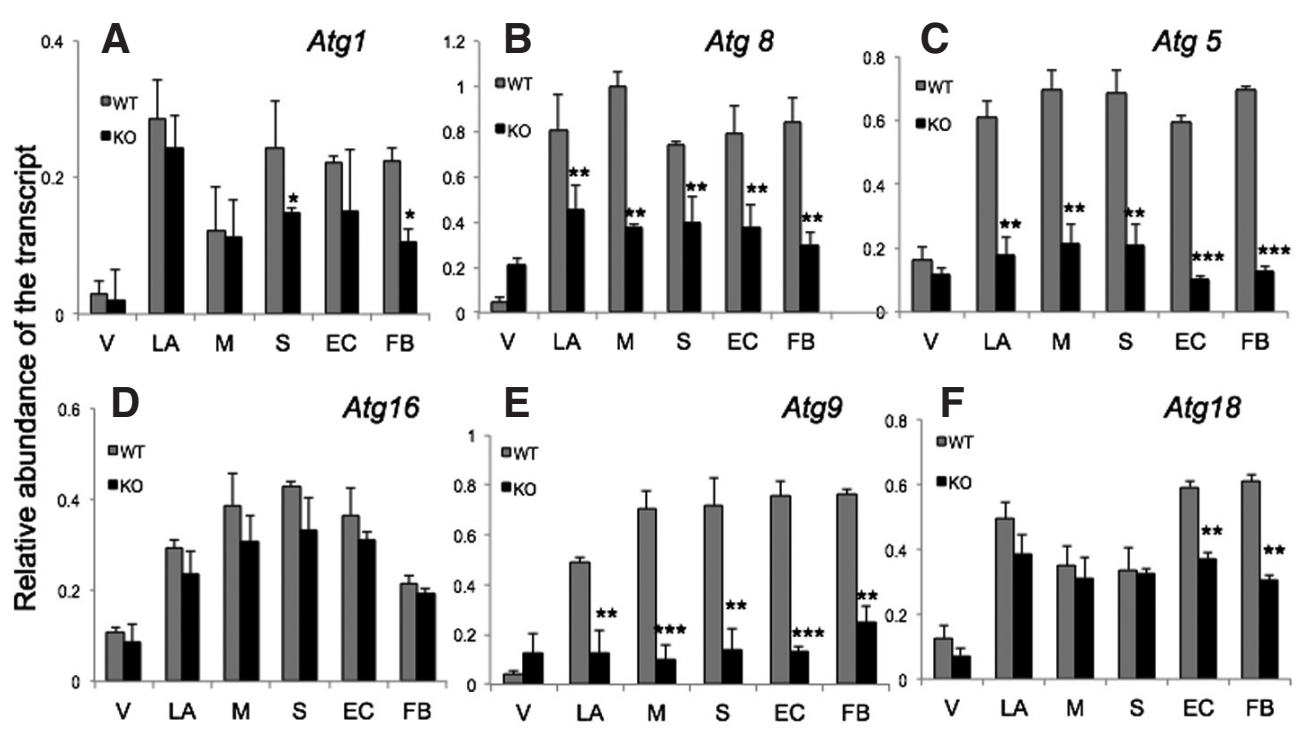
were observed during development of sir2D- cells. We observed a decrease in the Atg1 (involved in the induction of autophagy) expression (Fig. 7A), Atg8 (an autophagosome marker, Fig. 7B), Atg5 and Atg16 (from the ubiquitin-like conjugation system, Fig. 7C and D) Atg9 and Atg18 (involved in membrane trafficking, Fig. $7 \mathrm{E}$ and $\mathrm{F}$ ) expression in sir2D cells throughout development. Results show that Sir2D regulates the genes involved in the process of autophagy.

\section{sir2D - cells show reduced autophagy}

We wanted to determine whether DdSir2D was involved in autophagy. To this end, we transfected both the Ax2 and sir2Dcells with either RFP-GFP-Atg8 or GFP-Tkt-1 which would allow monitor autophagic flux. RFP-GFP-Atg8 tandem probe allows both microscopic visualization and monitoring of autophagic flux. It is well known that RFP fluorescence is resistant to acidic $\mathrm{pH}$ of the lysosome whereas, GFP fluorescence gets quenched rapidly. Thus, the red-green puncta marks the early autophagosomes and the red puncta that lack green fluorescence marks the fusion of autophagosomes with lysosomes. The sir2D-cells showed lower red puncta after starvation or $\mathrm{NH}_{4} \mathrm{Cl}$ treatments (Fig. 8B and D) as compared to the control wild-type (Fig. 8A and C). We found an increase in the red puncta in the wild-type upon starvation when compared to the sir2 $D^{-}$cells. The sir2 ${ }^{-}$cells failed to activate autophagy in response to nutrient starvation. Further, autophagic flux was also performed based on proteolytic cleavage (Fig. 8E). We found a significant increase in the cleaved GFP levels (measured by Western blot) upon $\mathrm{NH}_{4} \mathrm{Cl}$ treatment in the wild-type cells but no such increase was observed in case of sir2D-cells confirming the decrease in autophagic flux. The confirmation of subcellular localization of the cytosolic protein GFP-Tkt-1 is shown in Supplementary Fig. S5. Our data suggests the possible role of Sir2D in autophagy.

\section{Discussion}

In the present study, we characterized Sir2D, a member of class III histone deacetylase from Dictyostelium discoideum and provide a link to autophagy. DdSir2D is the ortholog of human SIRT1, which is a NAD+ dependent deacetylase. We show that overexpression of Sir2D increased cell proliferation while sir2D' inhibited both cell proliferation as well as autophagic flux. The knowledge gained from this study would help highlight the evolutionary conservation of DdSir2D/SIRT1 functions and also reiterate that $D$. discoideum could be used as a model system to study sirtuin biology.

There are five sirtuins present in $D$. discoideum whose functions are still unknown. Based on its sequence similarity Sir2D possibly could be a homolog of human SIRT1. Similar to the observations made by Katayama and Yasukawa (2008), we too observed insignificant differences in the isolated prestalk and prespore cells collected from the migrating slugs. mRNA is differentially expressed showing preference for prespore/spore region of the other multicellular structures developed. Expression of sir2D throughout development suggests its possible role in various cellular functions.

SIRT1 is a nuclear protein (McBurney et al., 2013) and its ortholog DdSir2D (as a fusion protein) also show the same localization.
Fig. 8. Sir2D and reduced autophagic flux. Confocal images of (A) $A \times 2$ and (B) sir2D cells transformed with tandemlytagged RFP-GFP-Atg8 in control and after stimulus with starvation $/ \mathrm{NH}_{4} \mathrm{Cl}$. Atg8 puncta/cell was counted using NIS elements software in (C) Ax2 and (D) sir2D cells. A total 30-cells/individual experiment was analyzed and statistical significance was calculated using unpaired t-test. Black bars represent green puncta and white bars represent red puncta. [scale bar: $5 \mu \mathrm{m} ; n=5 ; \mathrm{p}$ value: $\left.{ }^{* * *}<0.001 ;{ }^{*}{ }^{*}<0.01 ; ;{ }^{*}<0.05\right]$. (E) One representative blot showing autophagic flux analysis by Western hybridization. Strains transfected with the marker GFP-Tkt-1 growing in HL5 were incubated with 0 or $100 \mathrm{mM} \mathrm{NH} 4 \mathrm{Cl}$. Extracts containing the same protein load were subjected to SDS-PAGE, transferred byWestern blot, and incubated with $\alpha$-GFP antibody to detect GFP-Tkt-1 and the cleaved GFP proteins. Actin is taken as loading control. [* ${ }^{*}$ nonspecific band].
A
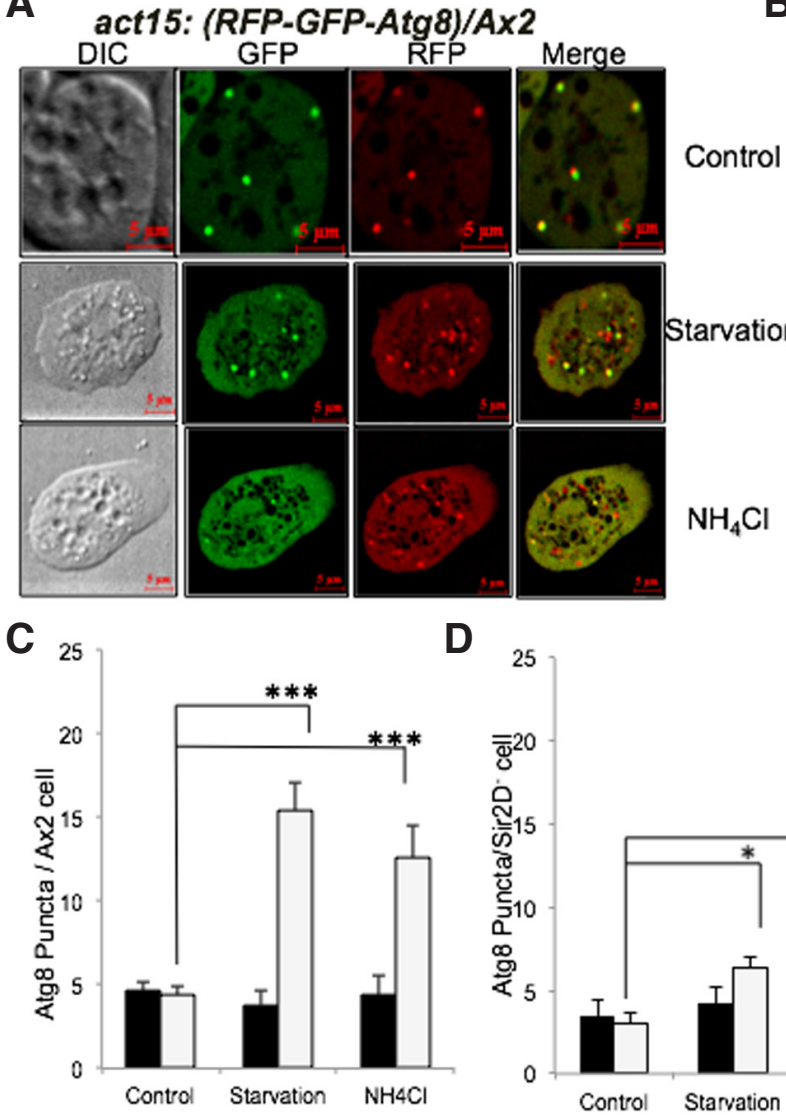

B act15: (RFP-GFP-Atg8)/sir2D-

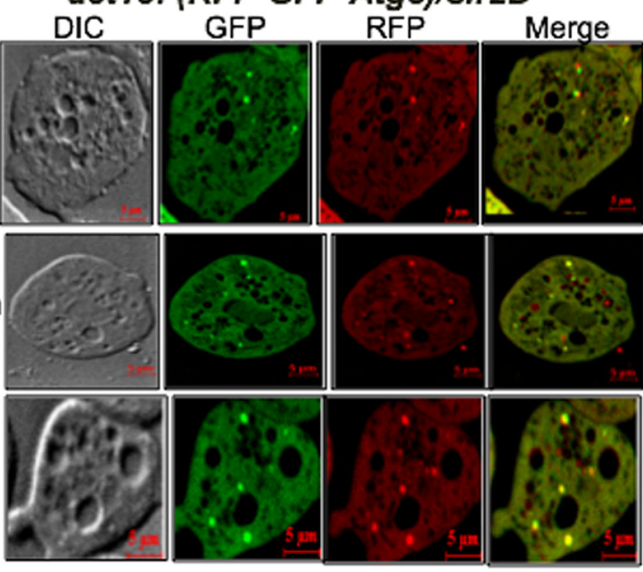

E

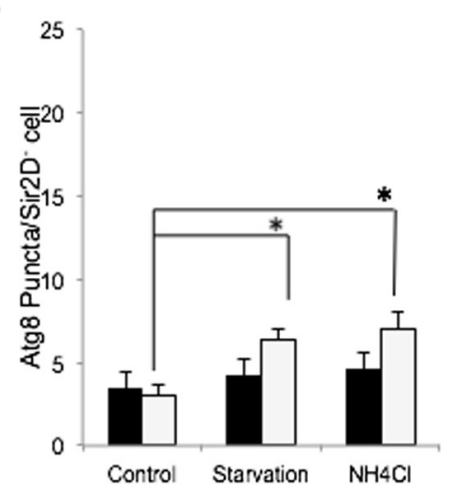

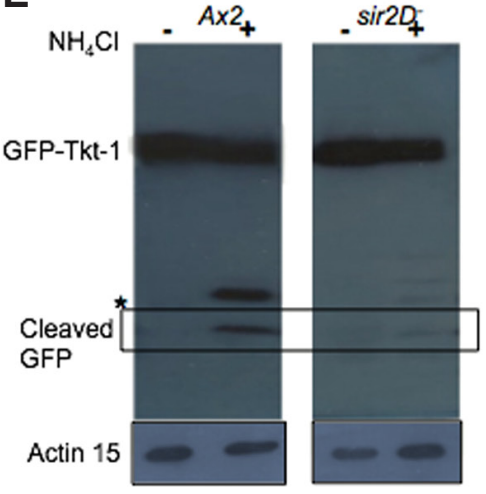


Dynamic changes in the subcellular localization of Drosophila Sir2 during embryonic development have also been reported. Thus, the subcellular localization of SIRT1 may change during development and in response to physiological and pathological stimuli (Tanno et al., 2007). Nucleus localized SIRT1 is also known to induce the expression of autophagy pathway components ( $\mathrm{Ng}$ and Tang 2013), which also is observed in $D$. discoideum.

Deletion of sir2D reduced cell proliferation and developed multi-tipped phenotype, similar to what is observed with other Atgs mutants. Sir2D maybe involved in the control of cell cycle however, details remain to be elucidated regarding the mechanism of function. Results show that in the absence of Sir2D protein, aberrant phenotype developed which could be attributed to two possible reasons: (i) it is an autophagic mutant showing a typical multitipped phenotype and possibly upon starvation results in slower development with smaller aggregates (Calvo-Garrido et al., 2010); or/and (ii) there was alteration in early development with respect to cAMP signalling and cell-adhesion molecules.

There are molecular markers of autophagy that are used for monitoring autophagy process of which the most common one is Atg8/LC3 that becomes lipidated and attached to the autophagosome membrane. Since autophagy is a dynamic process we monitored the autophagic flux by taking two different approaches. In the present study, we found reduced autophagic flux in the sir2Dcells. In case of $D$. discoideum starvation promotes the activation of autophagy. Besides starvation, autophagy can be activated by a variety of stresses and there could be several signalling pathways that may be implicated in the induction of autophagy like TORC1, AMPK and PI3K. Involvement of Sir2D in autophagy is similar to that observed in many other organisms. Here, we are not sure if Sir2D directly inactivates TORC1 complex to induce autophagy or there are any mediators. More work is required to determine the signalling pathways involved in autophagy. Expression of genes involved in various stages of autophagy process was reduced during development. Atg1 that plays a central role in the regulation of autophagy by integrating signals largely from nutrient stress was reduced, especially during later stages of development. In general, most of the Atg mutants studied (Luciani et al., 2011) in this organism show a multi-tipped structure leading to abnormal fruiting body formation. Observations of multi-tipped phenotype but later on a normal development of sir2D cells suggest that other sirtuins may cause partial redundancy of the phenotypes. This still needs to be investigated.

The second mechanism that may be possible is alteration in the cAMP signalling and relay during early development. Four major determinants regulating territory sizes in $D$. discoideum include cell number and their size in the aggregate, counting mechanism, cellcell adhesion and cAMP signal strength (Jang and Gomer 2008). Presently our studies indicate increased cAMP signalling during early development as the components ( $a c a A, p d s A$ and $\operatorname{car} A$ ) required for CAMP signalling and relay were found to increase. At the same time, the cell adhesion molecules cad $A$ and $c s A$ was also increased which could help hold the smaller aggregates tightly. During the process of development we found regA expression to increase suggesting that cAMP signalling must be regulated towards bringing about normalcy, so that development could proceed as in the wild-type. Our results suggest that it may not be the cAMP signalling which was responsible for this phenotype. The mutant sir2D cells show impaired autophagy. Strain mutated in genes involved in this process show a developmental phenotype including impaired aggregation, the formation of multi-tipped aggregates and small fruiting bodies. Therefore, we think the formation of small aggregates could be related to impaired autophagy in response to starvation.

Sir2D is involved in the regulation of a signalling molecule that is secreted out or is membrane bound, as a small percentage of wild-type cells were able to rescue the developmental defects observed in sir2 $D^{-}$cells. We found that mixing sir2 $D^{-}$cells with as little as $10 \%$ wild-type was able to rescue the observed developmental defects. The sir2 $D^{-}$cells in the chimeras preferentially showed biasness towards stalk pathway and also reduced ability to form spore cells. It could be suggested that the wild-type cells may provide sufficient quantities of a secreted molecule to allow the rescue of developmental defects observed with sir2D- cells. Since the prestalk cells initiate the process of culmination, we believe that the sir2D cells must be defective in spore cell differentiation rather than stalk cell differentiation. Reduced expression of cAMP regulated genes $p s p A$ and $e c m A O$ genes in multicellular structures formed by sir2D cells were also observed.

Induction of $D$. discoideum require atleast two successive signals: the first being starvation /cAMP to induce autophagy and secondly the differentiation-inducing factor (DIF) to lead them (Giusti et al., 2009) towards autophagic cell death (ACD). If autophagy were required for $A C D$ then a mutation in the sir2D-would

\section{TABLE 1}

\section{LIST OF OLIGONUCLEOTIDES USED IN THIS STUDY. SEQUENCES IN BOLD LETTERS MARK THE RESTRICTION ENZYME SITE}

\begin{tabular}{|c|c|c|}
\hline Gene name & primer & 5 ' to 3 ' sequence \\
\hline In situ & $\begin{array}{l}\text { FP } \\
\text { RP }\end{array}$ & $\begin{array}{l}\text {-ACGCGGATCCAATAAGAGATCTTTAGAAAATAATGAACTAAAC- } \\
\text {-ACGCCTCGAGTCCATTTAACTTTATTTAATAAATCTTG- }\end{array}$ \\
\hline $\begin{array}{l}\text { promoter of } \\
\operatorname{sir} 2 D\end{array}$ & $\begin{array}{l}\text { FP } \\
\text { RP }\end{array}$ & $\begin{array}{l}\text {-ACGCTCTAGAATCCCAGTTTCCCAATTGTAG- } \\
\text {-CGCGGATCCTCTCTTATTCATATCTTATTCTTTTTC- }\end{array}$ \\
\hline $\begin{array}{l}\text { sir } 2 D \text { prom } \\
+ \text { ORF }\end{array}$ & $\begin{array}{l}\text { FP } \\
\text { RP }\end{array}$ & $\begin{array}{l}\text {-ACGCTCTAGAATCCCAGTTTCCCAATTGTAG- } \\
\text {-CGCGGATCCCCATTTAACTTTATTTAATAAATCTTG- }\end{array}$ \\
\hline $\operatorname{sir} 2 D^{\mathrm{OE}}$ & $\begin{array}{l}\text { FP } \\
\text { RP }\end{array}$ & $\begin{array}{l}\text {-ACGCGGATCCAATAAGAGATCTTTAGAAAATAATGAACTAAAC- } \\
\text {-ACGCCTCGAGTCCATTTAACTTTATTTAATAAATCTTG- }\end{array}$ \\
\hline$a c a A$ & $\begin{array}{l}\text { FP } \\
\text { RP }\end{array}$ & $\begin{array}{l}\text {-AGTACACCACATAATAATAATCAT- } \\
\text {-CTCTGGAATTACAATATCTCTCTT- }\end{array}$ \\
\hline carA & $\begin{array}{l}\text { FP } \\
\text { RP }\end{array}$ & $\begin{array}{l}\text {-TGTATGGCAGTGTTGATTGGT- } \\
\text {-ATGGTGATGGATTGTTATTGT- }\end{array}$ \\
\hline$p d s A$ & $\begin{array}{l}\text { FP } \\
\text { RP }\end{array}$ & $\begin{array}{l}\text {-ATGGCATTAAATAAAAAATT- } \\
\text {-TTAAATACAAATTGGATCACC- }\end{array}$ \\
\hline cadA (gp24) & $\begin{array}{l}\text { FP } \\
\text { RP }\end{array}$ & $\begin{array}{l}\text {-TCTGTTGATGCAAATAAGTAAA- } \\
\text {-ATAGTCATATGGTGTATGTGTTTG- }\end{array}$ \\
\hline $\operatorname{cs} A(g p 80)$ & $\begin{array}{l}\text { FP } \\
\text { RP }\end{array}$ & $\begin{array}{l}\text {-GTGAACGACTCTATTAACTCTGCT- } \\
\text {-AGTTGGAGTGTCTGGAATTGTATA- }\end{array}$ \\
\hline$g b f A$ & $\begin{array}{l}\text { FP } \\
\text { RP }\end{array}$ & $\begin{array}{l}\text {-CCATTACCATTACCATCTATA- } \\
\text {-TGATGGTGATGGTGTATTACT- }\end{array}$ \\
\hline $\operatorname{reg} A$ & $\begin{array}{l}\text { FP } \\
\text { RP }\end{array}$ & $\begin{array}{l}\text {-TCAATGGTCATAATAATGGATTTG- } \\
\text {-CACATACAAGAGATTAATAGAGCG- }\end{array}$ \\
\hline Atgl & $\begin{array}{l}\text { FP } \\
\text { RP }\end{array}$ & $\begin{array}{l}\text {-ATGAAACGAGTAGGAGAT- } \\
\text {-TAATAGGTCTGGTACTGAACC- }\end{array}$ \\
\hline $\operatorname{Atg} 8$ & $\begin{array}{l}\text { FP } \\
\text { RP }\end{array}$ & $\begin{array}{l}\text {-ATGTATCAAGCTTTAAAAACGACCAC- } \\
\text {-TTATAAATCACTACCAAAAGTATTTTCACC- }\end{array}$ \\
\hline $\operatorname{Atg} 5$ & $\begin{array}{l}\text { FP } \\
\text { RP }\end{array}$ & $\begin{array}{l}\text {-ATGTCATCATTTGACGAA- } \\
\text {-GGGTATGATTGGAAATGAAC- }\end{array}$ \\
\hline $\operatorname{Atg} 16$ & $\begin{array}{l}\text { FP } \\
\text { RP }\end{array}$ & $\begin{array}{l}\text {-ATGTTTTCATCACAAAATAA- } \\
\text {-TCTTTCAACTAAACTACTA- }\end{array}$ \\
\hline $\operatorname{Atg} 9$ & $\begin{array}{l}\text { FP } \\
\text { RP }\end{array}$ & $\begin{array}{l}\text {-ATGTCACATGAAGATAGAGG- } \\
\text {-TCATAAATCTACGTGATAAT- }\end{array}$ \\
\hline $\operatorname{Atg} 18$ & $\begin{array}{l}\text { FP } \\
\text { RP }\end{array}$ & $\begin{array}{l}\text {-ATGAATGTTGGAGGTAAATT- } \\
\text {-TACTATGAATGATTGCAGGT- }\end{array}$ \\
\hline ig7 & $\begin{array}{l}\text { FP } \\
\text { RP } \\
\text { RP }\end{array}$ & $\begin{array}{l}\text {-GGATTCTGCAAAATGGCAAC- } \\
\text { - GTCCTCTCGTACTAAAGGAAGG- } \\
\text {-ACGCCTCGAGTCCATTTAACTTTATTTAATAAATCTTG- }\end{array}$ \\
\hline
\end{tabular}


prevent autophagy as well as ACD. In our case autophagic flux was decreased and the severity of the phenotype observed was corrected in later developmental stages. ACD did occur suggesting that Sir2D may be required for induction of autophagy or possibly partial redundancy by other sirtuins in autophagy, which still needs to be investigated. We believe that the sir2D cells protect the cells during growth and early development till the other sirtuins can take over. It is also possible that sir2D may have additional autophagyindependent functions. More work is required to show if Sir2D has autophagy-independent functions.

\section{Materials and Methods}

\section{Cell culture and development}

Dictyostelium discoideum Ax2 (axenic strain) cells were grown and developed according to Gosain et al., (2012). Exponentially growing cultures were inoculated at a density of $\sim 2-3 \times 10^{5} \mathrm{cell} / \mathrm{ml}$ and counted in a haemocytometer for monitoring cell proliferations.

\section{Multiple sequence alignment and phylogenetic analysis}

Genomic DNA, cDNA and protein sequences of DdSir2D were obtained from online resource (http://www.dictybase.org). Protein sequences of known sirtuins were obtained from UniProt KB (www.uniprot.org). Simple Modular Architecture Research Tool, SMART (http://SMART.embl-heidelberg.de) was used to deduce the domain architectures of the proteins. DdSir2D orthologs were searched using Basic Local Alignment Search Tool (BLASTp) at NCBI (http://blast.ncbi.nlm.nih.gov/Blast.cgi), Uniprot and dictyBase. Multiple alignments were performed using ClustalW2 at EBI server (http://www.ebi. ac.uk/Tools/clustalw2/). PHYLIP package (Phylogeny Inference Package, version 3.68) was used to construct Neighbor joining (NJ) phylogenetic trees with 1000 bootstrap replicates to create a consensus tree.

\section{RNA detection by RT-PCR and in situ hybridization}

RT-PCR: This was performed using Trizol reagent (Thermo Fisher Scientific) as described in Gosain et al., (2012) and amplified (Table 1). The cycle numbers used for amplification were within the linear range and ig7 was used as an internal control.

In situ hybridization: The spatial expression pattern of $D d s i r 2 D$ transcript was studied by in situ hybridization with DIG-labelled RNA probe (Gosain et al., 2012). A 1624 bp region of the Ddsir2D gene from the coding region (4-1790 bp) of the genomic DNA was cloned in the vector pBSIISK+ as an Xhol and BamHI fragment (Table 1). The construct was restricted with BamHI to yield template for antisense probe synthesis by T7 RNA polymerase and Xhol to yield template for sense probe synthesis by T3 RNA polymerase. Sense and antisense probes were hydrolysed due to their large sizes.

\section{Construction of reporter and expression vectors}

Eyfp reporter construct: Full length Ddsir2D gene was PCR amplified (Table 1) from the Ax2 genomic DNA (4-1790 bp) and expressed as a fusion protein with the enhanced yellow fluorescent protein (Eyfp) at the C-terminal. The constitutive promoter, actin 15 was used for driving its expression.

Creating [sir2D/ubi-lacZ] construct: The putative promoter of $D d s i r 2 D$ was used to drive the expression of the labile reporter gene $\beta$-galactosidase (ubi-lacZ). A region including 573 bp 5' to sir2D ORF (which hosts the putative promoter region) and the first 12 bases of the ORF was amplified (Table 1). This amplicon was used to replace the ecmAO promoter (Xbal and Bglll digestion) in $\mathrm{p}[\mathrm{ecmAO} / \mathrm{ubi}$-lacZ] vector.

Creating [sir2D/sir2D-ubi-lacZ] construct: The putative promoter with ORF region (2351bp) of $D d s i r 2 D$ was amplified (Table 1 ). The product was restricted with $\mathrm{Xbal}$ and $\mathrm{BamHI}$ and ligated into the vector upstream and in frame with the ubi-lacZ ORF sequence of $\mathrm{p}$ [ecmAO/ubi-lacZ] vector, replacing ecmAO promoter to drive the expressions of lac $Z$ (labile-gal) and generate $\mathrm{p}[$ sir2D/sir2D-ubi-lacZ].

Individual plasmids were transformed into Ax2 cells by electroporation and the transformants were selected for growth at $40 \mu \mathrm{g} / \mathrm{ml} \mathrm{G418}$ for the expression of reporter gene.

LacZ reporter constructs: $A \times 2$ and the mutant of interest [Ax2/sir2D-] were transformed with [ecmB/lacZ], [ecmAO/lacZ] or [pspA/lacZ] reporter constructs (Gosain et al., 2014) and selected at $40 \mu \mathrm{g} / \mathrm{ml} \mathrm{G418.}$

Histochemistry of $\beta$-galactosidase activity in developmental structures: $X$-gal staining of developing structures was carried out as described in Gosain et al.,2012).

\section{Disruption of the sir2D gene by homologous recombination}

To prepare a $D d s i r 2 D$ gene disruption construct, two DNA fragments of the sir2D gene comprising nucleotides from 85-1001 bp (as fragment BamHI/EcoRI) and from 1040-1757 (as fragment Xbal/BamHI) were amplified. These fragments were three-point ligated with Xbal and EcoR digested pUC101 vector (Sutoh 1993). The construct was linearized with BamHI resulting in a pUC101 plasmid flanked with a 917 and 718 bp of 5 and 3' sir2D sequences, respectively. The linearized gene targeting DNA was introduced into Ax2 cells and selected for growth at $5 \mu \mathrm{g} / \mathrm{ml}$ blasticidin S. Transformed cells were screened for homologous recombination by various PCR reactions and confirmed by sequencing and RT-PCR (Supplementary Fig. S3 and Table S1). The knockouts were called [sir2D-/Ax2].

\section{Development of chimeric mixtures}

Cells of different genetic backgrounds were mixed and allowed to coaggregate to form chimeric structures. For examining the distribution of cells in chimeric developmental organisms, wild-type Ax2 cells were transformed with (act15/eyfp) (marked), and were mixed with unmarked [sir2D-/ Ax2] cells in varying ratios Gosain et al., (2014). The vice-versa mixings were also performed. The mixed populations were allowed to develop on non-nutrient agar plates and the images were collected on a microscope (Nikon AZ100) with both DIC and fluorescence. A minimum of 20 sporeheads for each ratio from 4 independent experiments was scored. Error bars indicate standard error.

\section{Microscopy}

Images of development were captured using Nikon (AZ100) stereozoom. Confocal microscopy (Andor Spinning Disc) was used for capturing single cell images. The camera was controlled by NIS elements and processed with Adobe Photoshop software.

\section{Analysis of autophagic flux}

Autophagic flux in wild-type and sir2D cells, both under control and induced conditions were analysed by confocal microscopy and proteolytic cleavage. Wild-type and sir2D cells were transfected with a construct expressing the autophagic marker RFP-GFP-Atg8 as described previously (Calvo-Garrido et al., 2011). After selection on G418, cells were induced by different stimuli: starvation (cells grown in HL5 were washed two times and incubated for $2 \mathrm{~h}$ in $\mathrm{KK}_{2}$ buffer) and treatment with the lysosomotropic agent, $\mathrm{NH}_{4} \mathrm{Cl}(100 \mathrm{mM}$ for $4 \mathrm{~h})$. Cells were visualized by confocal microscopy and Z-stacks were selected to get maximum projections covering the whole cell. The fluorescent puncta/cell was monitored using confocal microscopy. Additionally, the flux was also measured as described by Calvo-Garrido et al., (2011). Briefly, [act15/GFP-Tkt-1] construct was made and transformed into Ax2 cells (Supplementary Fig. S5; Table S1). Equal amounts of protein from control and induced $\left(\mathrm{NH}_{4} \mathrm{Cl}\right)$ cells were loaded onto a 10\% SDS-PAGE gel. Western hybridization (Gosain et al., 2012) to detect the cleaved GFP and GFP-Tkt-1 using $\alpha$-GFP antibody (Sigma-Aldrich) was carried.

\section{Acknowledgements}

SS thanks DST(SR/SO/BB-18/2008) and ICMR (54/41/CFP/GER/2011NCD-II) for research grants. $R L$ and PJ thank CSIR and DST-INSPIRE for fellowships, respectively.

\section{Author contributions}

$R L, P J$ and $M J$ performed the experiment and analysed the data. SS, 
$R L$ and PJ conceived and designed the experiments. AS and PKB provided materials and help write the manuscript along with SS, RL and PJ. All authors read and approved the final manuscript.

\section{References}

CALVO-GARRIDO J, CARILLA-LATORRE S, KUBOHARAY, SANTOS-RODRIGON, MESQUITA A, SOLDATI T, GOLSTEIN P, ESCALANTE R (2010). Autophagy in Dictyostelium: genes and pathways, cell death and infection. Autophagy 6:686-701.

CALVO-GARRIDO J, CARILLA-LATORRE S, MESQUITAA, ESCALANTE R (2011). A proteolytic cleavage assay to monitor autophagy in Dictyostelium discoideum. Autophagy 7: 1063-1068.

COLETTA A, PINNEY WJ, SOLÍS WYD, MARSH J, PETTIFER RS, ATTWOOD KT (2010). Low-complexity regions within protein sequences have position-dependent roles. BMC Systems Biology 4: 43.

DALI-YOUCEF N, LAGOUGE M, FROELICH S, KOEHL C, SCHOONJANS K, AUWERX J (2007). Sirtuins: the 'magnificent seven', function, metabolism, and longevity. Ann Med 39: 335-345.

FINKEL T, DENG C, MOSTOSLAVSKY R (2009). Recent progress in the biology and physiology of sirtuins. Nature 460: 587-591.

FRYE RA (2000). Phylogenetic classification of prokaryotic and Eukaryotic Sir2-like proteins. Biochem Biophys Res Comm 273: 793-798.

GOSAIN A, LOHIA R, SHRIVASTAVA A, SARAN S (2012). Identification and characterization of peptide: $\mathrm{N}$ - glycanase from Dictyostelium discoideum. BMC Biochemistry 13: 9.

GOSAIN A, SHRIVASTAVAA, SARAN S (2014). Peptide: $\mathrm{N}$ - glycanase is expressed in prestalk cells and plays a role in the differentiation of prespore cells during development of Dictyostelium discoideum. Indian J Exp Biol 52: 197-206.

GUARENTE L (2000). Sir2 links chromatin silencing, metabolism, and aging. Genes Dev 14: 1021-1026.

GUISTI C, TRESSE E, LUCIANI MF, GOLSTEIN P (2009). Autophagic cell death: Analysis in Dictyostelium. Biochim Biophys Acta1793: 1422-1433.

HE C, KLIONSKY DJ (2009). Regulation mechanisms and signaling pathways of autophagy. Annu Rev Genet 43: 67-93.

JANG W, RH GOMER (2008). Combining experiments and modeling to understand size regulation in Dictyostelium discoideum. JR Soc Interface 5: S49-S58.

KATAYAMA T, YASUKAWA H (2008). Developmental and spatial expression of sir2 genes in the cellular slime mold Dictyostelium discoideum. Microbes Environ 23: 40-43.

KESSIN RH (2001). Dictyostelium- evolution, cell biology and the development of multicellularity. (Cambridge Univ Press).
KONIJN TM, RAPER KB (1961). Cell aggregation in Dictystelium discoideum. Dev Biol 3: 725-756.

LEE HI, CAO LIU, MOSTOSLAVSKY R, LOMBARD DB, LIU J, BRUNS NE, TSOKOS M, ALT FW, FINKEL T (2008). A role for the NAD-dependent deacetylase Sirt1 in the regulation of autophagy. Proc Natl Acad Sci USA 105: 3374-3399.

LI X, KAZGAN N (2011). Mammalian sirtuins and energy metabolism. Int. J Biol SC 7: 575-587.

LUCIANI MF, GIUSTIC, HARMSB, OSHIMAY, KIKUCHIH, KUBOHARAY, GOLSTEIN P (2011). Atg1 allows second-signaled autophagic cell death in Dictyostelium. Autophagy 7: 501-508.

MARTÍNEZ-REDONDO P, VAQUERO A (2013). The diversity of histone versus nonhistone sirtuin substrates. Genes Cancer 4: 148-163.

MCBURNEY MW, YANG X, JARDINE K, HIXON M, BOEKELHEIDE K, WEBB JR, LANSDORP PM, LEMIEUX M (2003). The mammalian SIR2-alpha protein has a role in embryogenesis and gametogenesis. Mol Cell Biol 23: 38-54.

MCBURNEY MW, CLARK-KNOWLES KV, CARON AZ, GRAY DA (2013). SIRT1 is a highly networked protein that mediates the adaptation to chronic physiological stress. Genes Cancer 4: 125-134.

MICHISHITA E, PARK JY, BURNESKIS JM, BARRETT JC, HORIKAWA I (2005). Evolutionarily conserved and non-conserved cellular localizations and functions of human SIRT proteins. Mol Biol Cell 16: 4623-4635.

NAKAGAWAT, GUARENTE L (2011). Sirtuins at a glance. J Cell Science 124:833-838.

NGF, TANG BL (2013). Sirtuins'modulation of autophagy. J Cell Physiol228:2262-2270.

OTTO GP, WUMY, KAZGANN, ANDERSON OR, KESSINRH (2003). Macroautophagy is required for multicellular development of the social amoeba Dictyostelium discoideum. J Biol Chem 278: 17636-17645.

OTTO GP, WU MY, KAZGAN N, ANDERSON OR, KESSIN RH (2004). Dictyostelium macroautophagy mutants vary in the severity of their developmental defects. $J$ Biol Chem 279: 15621-15629.

SUTOH K (1993). A transformation vector for Dictyostelium discoideum with a new selectable marker Bsr. Plasmid 30: 150-154.

TANNO M, SAKAMOTO J, MIURA T, SHIMAMOTO K, HORIO Y (2007). Nucleocytoplasmic shuttling of the $\mathrm{NAD}^{+}$-dependent histone deacetylase SIRT1. J Biol Chem 282: 6823-6832.

TUNG SM, UNAL C, LEY A, PENA C, TUNGGAL B, NOEGEL AA, KRUT O, STEINERT M, EICHINGER L (2010). Loss of Dictyostelium ATG9 results in a pleiotropic phenotype affecting growth, development, phagocytosis and clearance and replication of Legionella pneumophila. Cell Microbiol 12: 765-780.

VASSILOPOULOS A, FRITZ SK, PETERSEN RD, GIUS D (2011). The human sirtuin family: Evolutionary divergences and functions. Human Genomics 5: 485-496. 


\section{Further Related Reading, published previously in the Int. J. Dev. Biol.}

Cloning, expression and characterization of the ornithine decarboxylase gene from Dictyostelium discoideum

Rishikesh Kumar, Sheikh Rafia and Shweta Saran

Int. J. Dev. Biol. (2014) 58: 669-676

http://dx.doi.org/10.1387/ijdb.140174ss

The Dictyostelium prestalk inducer DIF-1 directs phosphorylation of a bZIP transcription factor

Yoko Yamada, Yuzuru Kubohara, Haruhisa Kikuchi, Yoshiteru Oshima, Hong-Yu Wang, Susan Ross and Jeffrey G. Williams Int. J. Dev. Biol. (2013) 57: 375-381

http://dx.doi.org/10.1387/ijdb.130046jw

\section{Peptide signaling in Hydra}

Toshitaka Fujisawa and Eisuke Hayakawa

Int. J. Dev. Biol. (2012) 56: 543-550

http://dx.doi.org/10.1387/ijdb.113477tf

Bimodal distribution of motility and cell fate in Dictyostelium discoideum

Pavana Goury-Sistla, Vidyanand Nanjundiah and Gopal Pande

Int. J. Dev. Biol. (2012) 56: 263-272

http://dx.doi.org/10.1387/ijdb.113384ps

Synergy between two transcription factors directs gene expression in Dictyostelium tiporganiser cells

Hong Yu Wang and Jeffrey G. Williams

Int. J. Dev. Biol. (2010) 54: 1301-1307

http://dx.doi.org/10.1387/ijdb.103141hw

Identification of a target for CudA, the transcription factor which directs formation of the Dictyostelium tip organiser

Hong-Yu Wang and Jeffrey G. Williams

Int. J. Dev. Biol. (2010) 54: 161-165

http://dx.doi.org/10.1387/ijdb.082723hw

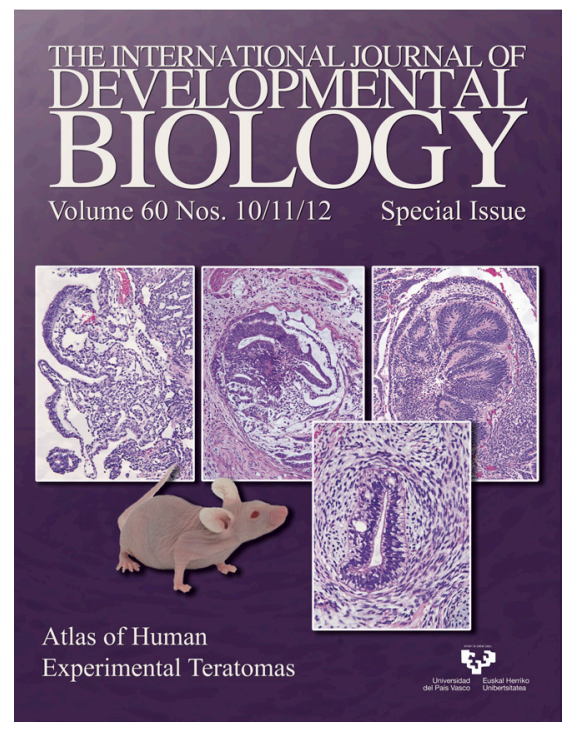

5 yr ISI Impact Factor $(2013)=2.879$
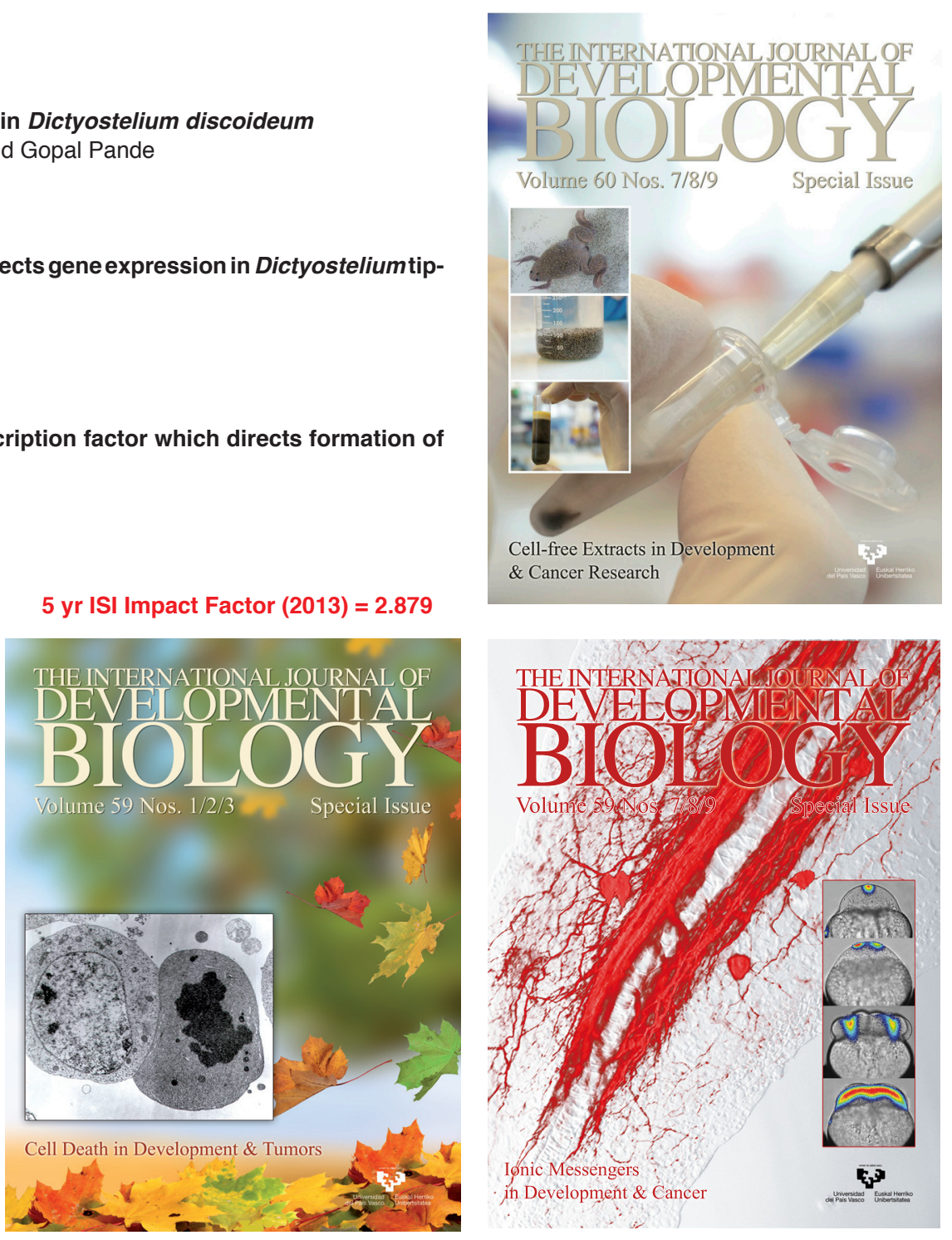